\title{
Generalized entropies in quantum and classical statistical theories
}

\author{
M. Portesi ${ }^{1}$, F. Holik ${ }^{1}$, P.W. Lamberti ${ }^{2}$, G.M. Bosyk ${ }^{1}$, G. Bellomo ${ }^{3}$ and S. Zozor ${ }^{4}$ \\ ${ }^{1}$ Instituto de Física La Plata, UNLP, CONICET, \\ Facultad de Ciencias Exactas, 1900 La Plata, Argentina \\ ${ }^{2}$ Facultad de Matemática, Astronomía, Física y Computación (FAMAF), UNC, CONICET, Córdoba, Argentina \\ ${ }^{3}$ CONICET-Universidad de Buenos Aires, Instituto de Investigación \\ en Ciencias de la Computación (ICC), Buenos Aires, Argentina and \\ ${ }^{4}$ Laboratoire Grenoblois d'Image, Parole, Signal et Automatique (GIPSA-Lab), CNRS, Saint Martin d'Hères, France
}

(Dated: February 26, 2018)

\begin{abstract}
We study a version of the generalized $(h, \phi)$-entropies, introduced by Salicrú et al, for a wide family of probabilistic models that includes quantum and classical statistical theories as particular cases. We extend previous works by exploring how to define $(h, \phi)$-entropies in infinite dimensional models.
\end{abstract}

\section{INTRODUCTION}

The concept of entropy has been widely used in the physics literature. But it has also been applied in information theory. An important example of a recent development combining both fields of research is given by quantum information theory [1]. In this field, quantum versions of information measures play a key role. The quantum counterpart of Shannon entropic measure is the von Neumann entropy [2 [4]. Also other measures have been adapted to the quantum realm in different contexts [5-10]. Entropic measures are important in several fields of research. They find applications in the study of:

- uncertainty measures (as is the case in the study of uncertainty relations [11 13]),

- different formulations of the MaxEnt principle [14 17],

- entanglement measuring and detection [18 24],

- measures of mutual information [25, 28, 32]

- the theory of quantum coding and quantum information transmission [4, 10, 29].

In the theory of classical information measures, Salicrú $(h, \phi)$-entropies [30] are, up to now, the most generalized extension containing the Shannon [31], Rényi [5] and Tsallis [6] entropies as particular examples.

A finite dimensional quantum version of the $(h, \phi)$-entropies was advanced and thoroughly studied in 33]. A generalization of the $(h, \phi)$-entropies to arbitrary finite dimensional probabilistic models was introduced in [34] (see [15, [35, 36] for generalizations of more restricted families of entropic measures to different frameworks). In this short paper we extend the previous definitions of $(h, \phi)$-entropies so as to include infinite dimensional models.

The paper is organized as follows. In Section [I we introduce preliminary notions of generalized probabilistic models and decomposition theory. In Section III we discuss the classical formulation of $(h, \phi)$-entropies and provide a definition of quantum $(h, \phi)$-entropies that includes infinite dimensional models. In Section IV we define $(h, \phi)$ entropies for probabilistic theories whose states form compact convex sets. Section $\mathrm{V}$ is devoted to some concluding remarks.

\section{PROBABILISTIC MODELS}

The description of quantum mechanical systems makes use of a family of probabilistic models that can be radically different from those originated in classical statistical theories. It is easy to show that both quantum and classical state spaces are convex sets [34]. Indeed, this result is much more general: in the approach to physical theories based on von Neumann algebras [43, 46] the sets of states are also convex. The canonical example of a von Neumann algebra is given by the set $\mathcal{B}(\mathcal{H})$ of bounded operators acting on a separable Hilbert space $\mathcal{H}$. Due to von Neumann double commutant theorem [46] 1 , it is possible to define a von Neumann algebra as a $*$-subalgebra $\mathbb{2}^{2} \mathcal{W} \subseteq \mathcal{B}(\mathcal{H})$ satisfying

\footnotetext{
${ }^{1}$ Given a subset $M \subseteq \mathcal{B}(\mathcal{H})$, the commutant of $M$ is defined as $M^{\prime}=\{A \in \mathcal{B}(\mathcal{H}) \mid A B-B A=0, \forall B \in M\}$.

${ }^{2}$ For bounded operators the $*$ operation means just taking the adjoint of a given operator (i.e., $A^{*}:=A^{\dagger}$ ). Thus, the condition "*-subalgebra" reads "is a subalgebra that is closed under the adjoint operation".
} 
$\mathcal{W}^{\prime \prime}=\mathcal{W}[47] . \mathcal{B}(\mathcal{H})$ is not the only example of a von Neumann algebra. By appealing to a dimension function, irreducible von Neumann algebras can be classified in terms of factors of Type I, II and III [43]. Only Type I factors appear in standard quantum mechanics: the set of matrices of a complex finite dimensional Hilbert space and $\mathcal{B}(\mathcal{H})$ (in the infinite dimensional case), are examples of Type I factors. But other factors may appear in the study of models of quantum mechanics involving infinitely many degrees of freedom (as is the case in quantum field theory [43, 47] and quantum statistical mechanics [37]). A commutative von Neumann algebra can be used to describe the algebra of observables of a classical probabilistic theory. States in general von Neumann algebras are defined in the standard way: a state $\nu: \mathcal{W} \longrightarrow \mathbb{C}$ is a continuous positive linear functional such that $\nu(\mathbf{I})=1$, with $\mathbf{I}$ the identity operator over $\mathcal{W}$. Positivity means that $\nu\left(A^{*} A\right) \geq 0$ for all $A \in \mathcal{W}$.

All von Neumann algebras are particular examples of $\mathrm{C}^{*}$-algebras (see, e.g., [37]). A $C^{*}$-algebra $\mathcal{M}$ is defined as a complex Banach algebra endowed with an $*$ involution satisfying $(\alpha a+\beta b)^{*}=\bar{\alpha} a^{*}+\bar{\beta} b^{*},(a b)^{*}=b^{*} a^{*}$ and $\left\|a a^{*}\right\|=\|a\|\left\|a^{*}\right\|$, for all $a, b \in \mathcal{M}$ and $\alpha, \beta \in \mathbb{C}$. All $\mathrm{C}^{*}$-algebras can be represented as $*$-subalgebras of $\mathcal{B}(\mathcal{H})$, closed under the norm operator topology. It is possible to show that, if the algebra $\mathcal{M}$ is unital, then, the set of states $\mathcal{C}(\mathcal{M})$ is convex and compact (in the weak ${ }^{*}$ topology [37, Chap.2]). Furthermore, due to the Krein-Milman theorem [39, Chap.1], the state space of a unital $\mathrm{C}^{*}$-algebra $\mathcal{M}$ is the weak* convex hull of its extreme points $\mathcal{E}(\mathcal{C}(\mathcal{M}))$.

Thus, in the rest of this paper, we will assume that the state spaces of the probabilistic models are compact convex subsets of a locally compact topological vector space. Notice that this assumption includes quantum theories (standard, statistical and relativistic) and classical theories as well, as particular cases. We denote by $\mathcal{C}$ the set of states of a given probabilistic model. The physical interpretation of the convexity assumption is that, given two states of the system, we should always be able to form a convex combination of them, representing a statistical mixture. Convex sets play a key role in the formal structure of quantum theory [49, 50]. The approach to quantum theories based in convex sets dates back to the works of B. Mielnik [48] and G. Ludwig [51] (at least). Recently, the operational approach based in convex sets has attracted much attention, related to the search of operational and informational axioms characterizing quantum theory (see for example [34, 36] and references therein).

The extreme points of the state space are termed pure states, while other states are known as mixed ones. As is well known, for the case of an arbitrary (compact) convex set of states $\mathcal{C}$ in finite dimensions, each state $\nu \in \mathcal{C}$ can be written as a convex combination of its extreme points. This is indeed the case in finite dimensional quantum and classical models [34]. In other words, for each state $\nu$, there exist a finite collection of extreme states $\left\{\nu_{i}\right\}_{1}^{n}$, such that $\nu$ can be written as

$$
\nu=\sum_{i=1}^{n} p_{i} \nu_{i} .
$$

where $p_{i} \geq 0$ and $\sum_{i=1}^{n} p_{i}=1$. The state space of a (finite-dimensional) classical model will be a $d$-dimensional simplex, which can be defined as the convex hull of $d+1$ linearly-independent points (defining a $d$-dimensional simplex). In such a simplex, a point can be expressed as a unique convex combination of its extreme points. It is remarkable that, for Abelian $\mathrm{C}^{*}$-algebras the state space is a simplex (see [37, Vol. 1, Chap. 4] and [39, Chap. 10] for more discussion on uniqueness of representing measures). Thus, the decomposition in terms of extreme points will also be unique. This characteristic feature of classical (commutative) theories no longer holds in quantum models. Indeed, even in the case of standard quantum mechanics of finite dimensional models, there are infinite ways to express a mixed state as a convex combination of extreme states.

In a more general theory described by a compact convex set $\mathcal{C}$, the decomposition of a given state in terms of the set $\mathcal{E}(\mathcal{C})$ of extreme points of $\mathcal{C}$ is more involved (see [37, 38] for details). Given $\omega \in \mathcal{C}$ the goal is to build a decomposition of the form

$$
\omega(a)=\int d \mu\left(\omega^{\prime}\right) \omega^{\prime}(a)
$$

where $\mu$ is a measure over $\mathcal{C}$ supported by the extremal points of $\mathcal{C}$ and $\omega$ is considered as a functional. This theory is related to the theory of barycentric decompositions in compact convex sets: given a normalized Radon measure in $\mathcal{C}$, its associated barycenter $b(\mu)$ will be given by

$$
b(\mu)=\int d \mu(\omega) \omega
$$

Given a $\mathrm{C}^{*}$-algebra $\mathcal{M}$ and a $\left(\right.$ weak $\left.^{*}\right)$ compact convex subset $\mathcal{S} \subseteq \mathcal{C}(\mathcal{M})$, it turns out that for every state $\omega \in \mathcal{S}$, 
there exists a maxima $3^{3}$ measure $\mu$, pseudosupported 4 in $\mathcal{E}(\mathcal{C}(\mathcal{M}))$ [35, 37], such that

$$
\omega=\int d \mu\left(\omega^{\prime}\right) \omega^{\prime}
$$

The above result is much more general: it is valid for arbitrary compact convex subsets of locally convex spaces (c.f. [39, Chap. 4] and [37, Vol. 1, Chap. 4]). As usual in noncommutative models, the above decomposition is not unique. For a given state $\omega$, we denote by $M_{\omega}(\mathcal{C})$ the set of all such measures.

\section{III. $(h, \phi)$-ENTROPIES}

In this section we discuss entropic measures in the context of standard quantum mechanics (i.e., we restrict our study to the case of Type I factors) and return to the general setting in Section IV. The $(h, \phi)$-entropies were introduced by Salicrú et al. [30] as follows:

Definition 1. Let us consider an $N$-dimensional probability vector $p=\left[p_{1} \cdots p_{N}\right] \in[0,1]^{N}$ with $\sum_{i=1}^{N} p_{i}=1$. The so-called $(h, \phi)$-entropies are defined as

$$
H_{(h, \phi)}(p)=h\left(\sum_{i=1}^{N} \phi\left(p_{i}\right)\right),
$$

where the entropic functionals $h: \mathbb{R} \mapsto \mathbb{R}$ and $\phi:[0,1] \mapsto \mathbb{R}$ are such that either: (i) $h$ is increasing and $\phi$ is concave, or (ii) $h$ is decreasing and $\phi$ is convex. In both cases, we restrict $\phi$ to be strictly concave/convex and $h$ to be strictly monotone, together with $\phi(0)=0$ and $h(\phi(1))=0$.

The family of $(h, \phi)$-entropies (5]) includes, as particular cases, the Shannon [31], Rényi [5], Havrda-Charvát-Tsallis [6, 52, 53], unified [54] and Kaniadakis [9] entropies. Dealing with the infinite dimensional context, the above definition naturally extends where the sum is then over $i \in \mathbb{N}$ provided the sum is finite (otherwise, by convention, the entropy is set to be infinite).

In reference [33], a quantum mechanical version of the $(h, \phi)$-entropies was introduced and some of their general properties were discussed. But this definition was restricted to finite dimensional quantum models. In what follows we advance a definition for the infinite dimensional case. We need to introduce first the following concepts. Let us denote the set of Hilbert-Schmidt operators acting on $\mathcal{H}$ by $\mathcal{B}_{H S}:=\left\{T \in \mathcal{B}(\mathcal{H}): \operatorname{Tr}\left(T^{2}\right)<\infty\right\}[50$. As is well known, the set $\mathcal{B}_{H S}$ endowed with the inner product $\left\langle T_{1}, T_{2}\right\rangle=\operatorname{Tr}\left(T_{2}^{\dagger} T_{1}\right)$ is a Hilbert space. For $T \in \mathcal{B}(\mathcal{H})$ the absolute value of $T$ is defined by $|T|=\left(T^{\dagger} T\right)^{\frac{1}{2}}$. The subspace formed by the trace class operators is defined as $\mathcal{B}_{1}(\mathcal{H})=\left\{T \in \mathcal{B}(\mathcal{H}):|T|^{\frac{1}{2}} \in \mathcal{B}_{H S}\right\}$. Quantum states can be defined as positive trace class operators of trace one (also called density operators). Now we can define the quantum $(h, \phi)$-entropies.

Definition 2. Let us consider a quantum system described by a density operator $\rho$ (i.e., a positive trace class operator of trace one) acting on a Hilbert space $\mathcal{H}$. The quantum $(h, \phi)$-entropies are defined as

$$
\mathbf{H}_{(h, \phi)}(\rho)=h(\operatorname{Tr} \phi(\rho))
$$

where the entropic functionals $h: \mathbb{R} \mapsto \mathbb{R}$ and $\phi:[0,1] \mapsto \mathbb{R}$ are such that either: (i) $h$ is strictly increasing and $\phi$ is strictly concave, or (ii) $h$ is strictly decreasing and $\phi$ is strictly convex. We impose $\phi(0)=0$ and $h(\phi(1))=0$ and we take the convention $\mathbf{H}_{(h, \phi)}(\rho)=+\infty$ whenever $\sum_{i \in \mathbb{N}} \phi\left(p_{i}\right)$ is not convergent. Here $\left\{p_{i}\right\}_{i \in \mathbb{N}}$ is the sequence of eigenvalues of the spectral decomposition of $\rho$, sorted in decreasing order and counted with their respective multiplicities.

The last convention of this definition is justified as follows. Every positive trace class operator $\rho$ of trace one admits a spectral decomposition of the form $\sum_{i \in \mathbb{N}} \lambda_{i} \mathbf{P}_{i}$, where $\left\{\mathbf{P}_{i}\right\}_{i \in \mathbb{N}}$ is a family of projection operators. Thus, $\phi(\rho)=$ $\sum_{i \in \mathbb{N}} \phi\left(p_{i}\right) \mathbf{P}_{i}$. But then, $\mathbf{H}_{(h, \phi)}(\rho)$ exists, only if $h(\operatorname{Tr}(\phi(\rho)))=h\left(\sum_{i \in \mathbb{N}} \phi\left(p_{i}\right)\right)<\infty$.

\footnotetext{
3 An order " $\leq "$ is introduced for the measures in $M_{+}(\mathcal{C})$ as follows: $\mu \leq \nu$, if and only if, $\mu(f) \leq \nu(f)$ for all real continuous convex functions. A measure $\mu$ is said to be maximal with respect to " $\leq$ " if, for all $\nu$ satisfying $\nu \geq \mu$, we have $\nu=\mu[37$, Vol. 1, Chap. 4]

${ }^{4}$ Given a compact convex set $\mathcal{C}$, a measure $\mu$ is pseudosupported by the set of its extreme points $\mathcal{E}(\mathcal{C})$, if for each Baire set $B \subseteq \mathcal{C}$ satisfying $B \cap \mathcal{E}(\mathcal{C})=\emptyset$, we have $\mu(B)=0$ [37, Vol. 1, Chap. 4].

${ }^{5}$ Notice that the spectral decomposition can be easily rewritten in terms of rank one projections as $\rho=\sum_{i \in \mathbb{N}} s_{i}\left|\phi_{i}\right\rangle\left\langle\phi_{i}\right|$, with $\sum_{i \in \mathbb{N}} s_{i}=$ $1, s_{i} \geq 0$ and $s_{i} \geq s_{i+1}$. This is known as the Schatten decomposition of $\rho$ (see, e.g., [35]).
} 
Regarding convergence in Definition 2, the following remarks are in order. Notice that $h\left(\sum_{i \in \mathbb{N}} \phi\left(p_{i}\right)\right)$ will be a convergent quantity -for all $h$ and $\phi$ - whenever the rank of $\rho$ is finite dimensional. In principle, even if infinite dimensional ranks are allowed, one may try to determine, given a particular choice of $h$ and $\phi$, the set of states $\rho$ for which the sum converges. Notice also that for important families of entropic functionals, $h\left(\sum_{i} \phi\left(p_{i}\right)\right)$ will be convergent for all states. As an example, consider the case of the Rényi entropies with entropic index greater than one. A detailed study of the convergence properties of the infinite dimensional $(h, \phi)$-entropies will be carried out elsewhere.

For finite dimensional $\mathcal{H}$, the Definition 2 reduces to the one introduced in [33]. Furthermore, the quantum $(h, \phi)$ entropy of a density operator $\rho$ equals the classical entropy of the sequence $p=\left\{p_{i}\right\}_{i \in \mathbb{N}}$ formed by its eigenvalues: $\mathbf{H}_{(h, \phi)}(\rho)=H_{(h, \phi)}(p)$.

An important notion for the rest of this work is that of majorization. Given two sequences $p=\left\{p_{i}\right\}_{i \in \mathbb{N}}$ and $q=\left\{q_{i}\right\}_{i \in \mathbb{N}}$ of positive real numbers sorted in decreasing order, we say that $q$ is majorized by $p$ (and we denote it by $q \preceq p$ ), if and only if, $\sum_{i=1}^{k} q_{i} \leq \sum_{i=1}^{k} p_{i}$ for all $k \in \mathbb{N}$ and $\sum_{i \in \mathbb{N}} q_{i}=\sum_{i \in \mathbb{N}} p_{i}$.

In what follows we make use of the integral form of the Jensen's inequality (c.f. [42]). Let $\mu$ be the Lebesgue measure, $f:[a, b] \longrightarrow \mathbb{R}$ be a Lebesgue-integrable function and $\phi$ a convex function. Then, for this case, Jensen's inequality reads

$$
\phi\left(\frac{1}{b-a} \int_{a}^{b} f(x) d x\right) \leq \frac{1}{b-a} \int_{a}^{b} \phi(f(x)) d x
$$

Assume that, for two sequences $p$ and $q$, we have that $p \succeq q$. Thus, $q=Q p$ with $Q_{i j}=\left|U_{i j}\right|^{2}$ for some unitary operator $U$ (see [41]). Due to the fact that $\sum_{j \in \mathbb{N}} Q_{i j}=1$, for each $i \in \mathbb{N}$, we can decompose the unit interval as

$$
[0,1]=\bigcup_{k=0}^{\infty}\left[\sum_{j=1}^{k} Q_{i j}, \sum_{j=1}^{k+1} Q_{i j}\right]
$$

(where we adopt the convention $\sum_{j=1}^{0} Q_{i j}=0$ ). Put in words: we write the unit interval as an infinite union of segments whose lengths are given by the sequence $\left\{Q_{i j}\right\}_{j \in \mathbb{N}}$. Define a step function $f:[0,1] \longrightarrow \mathbb{R}$ as $f(x)=p_{k+1}$ when $x \in\left[\sum_{j=1}^{k} Q_{i j}, \sum_{j=1}^{k+1} Q_{i j}\right)$ and $f(1)=0$. By construction, we have that $\int_{0}^{1} f(x) d x=\sum_{k \in \mathbb{N}} Q_{i k} p_{k}=q_{i}$ and $\int_{0}^{1} \phi(f(x)) d x=\sum_{k \in \mathbb{N}} Q_{i k} \phi\left(p_{k}\right)$. Thus, applying Jensen's inequality, we obtain

$$
\phi\left(q_{i}\right) \leq \sum_{k \in \mathbb{N}} Q_{i k} \phi\left(p_{k}\right)
$$

Summing over $i \in \mathbb{N}$, we have

$$
\sum_{i \in \mathbb{N}} \phi\left(q_{i}\right) \leq \sum_{i \in \mathbb{N}} \sum_{k \in \mathbb{N}} Q_{i k} \phi\left(p_{k}\right)=\sum_{k \in \mathbb{N}} \phi\left(p_{k}\right)
$$

Let us now invoke Theorem 4.1 of Ref. [40], that we reproduce here for the sake of completeness. Let $\mathcal{A}$ be the maximal Abelian von Neumann generated by the orthogonal set of rank-one projection operators $\left\{\left|e_{k}\right\rangle\left\langle e_{k}\right|\right\}_{k \in \mathbb{N}}$ and define the conditional expectation map $E: \mathcal{B}(\mathcal{H}) \longrightarrow \mathcal{A}$

$$
E(A)=\sum_{n \in \mathbb{N}}\left\langle e_{k}|A| e_{k}\right\rangle\left|e_{k}\right\rangle\left\langle e_{k}\right|
$$

Given $\left\{\lambda_{n}\right\}_{n \in \mathbb{N}}$, a decreasing sequence in $\ell^{1}$ with non-negative terms, let $\% 0 \lambda$ be the set of trace class operators possessing $\lambda$ as eigenvalue list. Then, Theorem 4.1 of [40] asserts that $E(\% \circ \lambda)$ consists of all positive trace-class operators $B \in \mathcal{A}$ whose eigenvalue list $\left\{p_{n}\right\}_{n \in \mathbb{N}}$ (arranged in decreasing order) is majorized by $\lambda$. Then, it follows that, if the density operator $\rho$ has an eigenvalue list $\lambda=\left\{\lambda_{n}\right\}_{n \in \mathbb{N}}$, the list formed by $p=\left\{\left\langle e_{n}|\rho| e_{n}\right\rangle\right\}_{n \in \mathbb{N}}$ (sorted in decreasing order) is majorized by $\left\{\lambda_{n}\right\}_{n \in \mathbb{N}}$. Thus, as we have seen above, for a convex function $\phi$ we have $\sum_{n \in \mathbb{N}} \phi\left(\left\langle e_{n}|\rho| e_{n}\right\rangle\right) \leq \sum_{n \in \mathbb{N}} \phi\left(\lambda_{n}\right)$. Remembering our convention for convex functions in Definition 2, we have 


$$
h\left(\sum_{n \in \mathbb{N}} \phi\left(\lambda_{n}\right)\right) \leq h\left(\sum_{n \in \mathbb{N}} \phi\left(\left\langle e_{n}|\rho| e_{n}\right\rangle\right)\right) .
$$

In other words, we obtain

$$
\mathbf{H}_{(h, \phi)}(\rho) \leq H_{(h, \phi)}(p) .
$$

A similar conclusion holds for the case of $h$ strictly increasing and $\phi$ strictly concave. It is interesting to compare inequality 13 with Proposition 5 of [33].

Due to the fact that the trace is invariant under arbitrary isometries (i.e., transformations implemented by operations satisfying $U^{\dagger} U=\sqrt{6}$ ), it is easy to check that:

Proposition 1. The quantum $(h, \phi)$-entropies are invariant under any isometric transformation $\rho \rightarrow U \rho U^{\dagger}$ where $U$ is an isometric operator:

$$
\mathbf{H}_{(h, \phi)}\left(U \rho U^{\dagger}\right)=\mathbf{H}_{(h, \phi)}(\rho) .
$$

Several properties of the family of entropies from Definition 2 were studied for the case of finite dimensional Hilbert spaces in [33]. The study of the properties in the infinite dimensional case is left for future work.

\section{ENTROPIES IN GENERALIZED PROBABILISTIC MODELS}

In this section, we introduce an extension of the definition of classical and quantum Salicrú entropies to a more general family of probabilistic theories.

\section{A. $(h, \phi)$-entropies in $\mathbf{C}^{*}$-algebras}

Let us first define our extension to $\mathrm{C}^{*}$-algebra models. We follow a strategy that is analogous to that of [35].

Definition 3. Given a $C^{*}$-algebra $\mathcal{M}$, for every $\omega \in \mathcal{C}(\mathcal{M})$, let:

$$
\begin{gathered}
D_{\omega}(\mathcal{C}(\mathcal{M})):=\left\{\mu \in M_{\omega}(\mathcal{C}(\mathcal{M})) \mid\right. \\
\left.\exists\left\{\mu_{k}\right\}_{k \in \mathbb{N}} \subset \mathbb{R}^{+} \text {and } \quad \phi_{k} \subset \mathcal{E}(\mathcal{C}(\mathcal{M})) \text { s.t. } \sum_{k \in \mathbb{N}} \mu_{k}=1 \text { and } \mu=\sum_{k \in \mathbb{N}} \mu_{k} \delta\left(\phi_{k}\right)\right\}
\end{gathered}
$$

where $\delta(\phi)$ is the Dirac measure centered at point $\phi$. Now, for $\mu \in D_{\omega}$ let

$$
H(\mu)=h\left(\sum_{k \in \mathbb{N}} \phi\left(\mu_{k}\right)\right)
$$

when the above sum converges and $H(\mu)=+\infty$ otherwise. Then, by imposing to the functions $h$ and $\phi$ the same conditions of Definition 圆, we define

$$
\mathbf{H}_{(h, \phi)}(\omega)=\left\{\begin{array}{l}
\inf \left\{H(\mu) \mid \mu \in D_{\omega}\right\} \\
+\infty \quad \text { if } D_{\omega}=\emptyset \\
+\infty \quad \text { if } \forall \mu \in D_{\omega}, H(\mu)=+\infty
\end{array}\right.
$$

\footnotetext{
${ }^{6}$ Notice that all unitary operators are isometries
} 
It is important to remark that all models of standard quantum mechanics are Type I factors (Type $\mathrm{I}_{n}$ for finite dimensional models and Type $\mathrm{I}_{\infty}$ for infinite dimensional ones), which are $\mathrm{C}^{*}$-algebras. For many measures, the above definition collapses into the one of standard quantum mechanics when restricted to Type I factors. Indeed, if $\mathcal{M}$ is a Type I factor, by Gleason's theorem [55], every state $\omega$ can be described by a positive trace class operator $\rho_{\omega}$ of trace one. For finite dimensional models, we reobtain the quantum $(h, \phi)$-entropies of [33]. For infinite dimensional Hilbert spaces, as is well known, the von Neumann entropy has the same minimization property (see for example [35]). Furthermore, Abelian $\mathrm{C}^{*}$-algebras are in correspondence to classical statistical models. Thus, our definition contains also an important class of classical models as particular cases.

\section{B. More general models}

We now discuss how to define the $(h, \phi)$-entropies in an arbitrary compact convex set $\mathcal{C}$, understood as the statespace of a generalized probabilistic model. We will combine the approach presented in [34] with the strategy used in Section IVA. Given a probabilistic model described by a compact convex set $\mathcal{C}$, let $\omega \in \mathcal{C}$ be a state. Denote by $M_{1}(\mathcal{C})$ to the set of normalized Radon measures [37, 38]. If $\omega$ is the barycenter of $\mathcal{C}$ with respect to measure $\mu$, we denote this by $\omega=b(\mu)=\int d \mu\left(\omega^{\prime}\right) \omega^{\prime}$. Define

$$
M_{\omega}(\mathcal{C})=\left\{\mu \mid \mu \in M_{1}(\mathcal{C}) \text { and } \omega=b(\mu)\right\}
$$

Now, in analogy to the procedure of Section III, we build the set $D_{\omega}(\mathcal{C})$, and proceed in a similar way as before.

Definition 4. Given a statistical theory whose state space is represented by a compact convex set $\mathcal{C}$, define

$$
\begin{gathered}
D_{\omega}(\mathcal{C}):=\left\{\mu \in M_{\omega}(\mathcal{C}) \mid\right. \\
\left.\exists\left\{\mu_{k}\right\}_{k \in \mathbb{N}} \subset \mathbb{R}^{+} \text {and } \phi_{k} \subset \mathcal{E}(\mathcal{C}) \text { s.t. } \sum_{k \in \mathbb{N}} \mu_{k}=1 \text { and } \mu=\sum_{k \in \mathbb{N}} \mu_{k} \delta\left(\phi_{k}\right)\right\}
\end{gathered}
$$

For $\mu \in D_{\omega}$, define

$$
H(\mu)=h\left(\sum_{k \in \mathbb{N}} \phi\left(\mu_{k}\right)\right)
$$

when the above sum converges and $H(\mu)=+\infty$ otherwise. Then, by imposing the same conditions on the functions $h$ and $\phi$ as in Definition 圆, we define

$$
\mathbf{H}_{(h, \phi)}(\omega)=\left\{\begin{array}{l}
\left.\inf \{H(\mu)) \mid \mu \in D_{\omega}\right\} \\
+\infty \quad \text { if } D_{\omega}=\emptyset \\
+\infty \quad \text { if } \quad \forall \mu \in D_{\omega}, \mathcal{H}(\mu)=\infty
\end{array}\right.
$$

In this way, we obtain a formal expression for the $(h, \phi)$-entropies in generalized probabilistic models.

It is important to notice that, given a state $\omega \in \mathcal{C}$, the set $D_{\omega}(\mathcal{C})$ can be used to define a notion of majorization in generalized probabilistic models in a similar way as in [34].

Definition 5. Suppose that there exists a discrete measure $\tilde{\mu}$ such that, for all $\mu \in D_{\omega}(\mathcal{C})$, if we put $\left\{\tilde{\mu}_{i}\right\}_{i \in \mathbb{N}}$ and $\left\{\mu_{i}\right\}_{i \in \mathbb{N}}$ in decreasing order, we have that $\sum_{i=1}^{k} \mu_{i} \leq \sum_{i=1}^{k} \tilde{\mu}_{i}$ for all $k$ and $\sum_{i \in \mathbb{N}} \mu_{i}=\sum_{i \in \mathbb{N}} \tilde{\mu}_{i}$. Then, by construction, we have that $\left\{\tilde{\mu}_{i}\right\}_{i \in \mathbb{N}}$ majorizes $\left\{\mu_{i}\right\}_{i \in \mathbb{N}}$ (and we write $\left\{\mu_{i}\right\}_{i \in \mathbb{N}} \preceq\left\{\tilde{\mu}_{i}\right\}_{i \in \mathbb{N}}$ ) for all $\mu \in D_{\omega}(\mathcal{C})$. In that case, we say that $\tilde{\mu}$ is the majorant of $D_{\omega}(\mathcal{C})$, and we call $\left\{\tilde{\mu}_{i}\right\}_{i \in \mathbb{N}}$ the spectra of $\omega$. Thus, if $\tilde{\mu}$ is the spectra of $\omega$ and $\tilde{\nu}$ is the spectra of $\sigma$, and we have $\left\{\tilde{\nu}_{i}\right\}_{i \in \mathbb{N}} \preceq\left\{\tilde{\mu}_{i}\right\}_{i \in \mathbb{N}}$, we then say that $\sigma \preceq \omega$ (i.e., $\omega$ majorizes $\sigma$ ).

\section{FINAL COMMENTS}

In this short paper, we have advanced a definition of the $(h, \phi)$-entropies for general probabilistic theories, extending previous definitions by including (possibly) infinite dimensional models. These examples include those of unital $\mathrm{C}^{*}$-algebras and more general compact convex sets. Associated to the above definitions, a natural definition of majorization for generalized probabilistic models arises (generalizing the definitions presented in [34]). A thorough study of the properties of these entropic measures is left for future work. 


\section{ACKNOWLEDGEMENTS}

MP, FH, PWL, GMB and GB acknowledge CONICET, UNLP and UBA (Argentina), and MP and PWL also acknowledge SECyT-UNC (Argentina) for financial support. SZ is grateful to the University of Grenoble-Alpes and CNRS (France). MP acknowledges an AUIP grant and warm hospitality at Universidad de Granada (Spain).

[1] M.A. Nielsen, I.L. Chuang, Quantum Computation and Quantum Information, Cambridge University Press, Cambridge, 2010 .

[2] J. von Neumann, Nachr. Ges. Wiss. Gottingen, Math.-Phys. Kl., 273-291 (1927).

[3] Holik, A. Plastino and M. Sáenz, Quantum Inf. Comput. 16 0115-0133 (2016).

[4] N. Watanabe, Phil. Trans. R. Soc. A 37420150240 (2016).

[5] A. Rényi, in Proc. Fourth Berkeley Symp. on Math. Statist. and Prob., Vol. 1, 547 (1961).

[6] C. Tsallis, J. Stat. Phys. 52479 (1988).

[7] N. Canosa, R. Rossignoli, Phys. Rev. Lett. 88170401 (2002).

[8] X. Hu, Z. Ye, J. Math. Phys. 47023502 (2006).

[9] G. Kaniadakis, Phys. Rev. E 66056125 (2002).

[10] G. Bellomo, G.M. Bosyk, F. Holik and S. Zozor, Sci. Rep. (in press).

[11] J.B.M. Uffink, Measures of Uncertainty and the Uncertainty Principle., Ph.D. thesis, University of Utrecht, Utrecht, The Netherlands, 1990. See also references therein.

[12] S. Zozor, G.M. Bosyk, M. Portesi, J. Phys. A 46465301 (2013).

[13] S. Zozor, G.M. Bosyk, M. Portesi, J. Phys. A 47495302 (2014).

[14] E.T. Jaynes, Probability Theory: The Logic of Science, Cambridge University Press, Cambridge, UK, 2003.

[15] C.A. Hein, Found. Phys. 9, 751-786 (1979).

[16] F. Holik and A. Plastino, J. Math. Phys. 53073301 (2012).

[17] F. Holik, C. Massri, and A. Plastino, Int. J. Geom. Methods Mod. Phys. 131650025 (2016).

[18] R. Horodecki, P. Horodecki, Phys. Lett. A 194147 (1994).

[19] S. Abe, A.K. Rajagopal, Physica A 289157 (2001).

[20] C. Tsallis, S. Lloyd, M. Baranger, Phys. Rev. A 63042104 (2001).

[21] R. Rossignoli, N. Canosa, Phys. Rev. A 67042302 (2003).

[22] I. Bengtsson, K. Życzkowski, Geometry of Quantum States: An Introduction to Quantum Entanglement, Cambridge University Press, Cambridge, 2006.

[23] Y. Huang, IEEE Trans. Inf. Theory 596774 (2013).

[24] K. Ourabah, A. Hamici-Bendimerad, M. Tribeche, Phys. Scripta 90045101 (2015).

[25] R.W. Yeung, IEEE Trans. Inf. Theory 431924 (1997).

[26] Z. Zhang, R.W. Yeung, IEEE Trans. Inf. Theory 441440 (1998).

[27] J. Cardy, J. Phys. A 28285402 (2013).

[28] D. Gross, M. Walter, J. Math. Phys. 54082201 (2013).

[29] R. Ahlswede, P. Löber, IEEE Trans. Inf. Theory 47474 (2001).

[30] M. Salicrú, M.L. Menéndez, D. Morales, L. Pardo, Communications in Statistics - Theory and Methods 22(7), 2015 (1993).

[31] C.E. Shannon, The Bell System Technical Journal 27, 623 (1948).

[32] N. Watanabe, Phil. Trans. R. Soc. A 37420150240 (2016).

[33] G.M. Bosyk, S. Zozor, F. Holik, M. Portesi and P.W. Lamberti, Quantum Inform. Process. 15 3393-3420 (2016).

[34] F. Holik, G.M. Bosyk and G. Bellomo, Entropy 17 7349-7373 (2015).

[35] M. Ohya, Rep. Math. Phys. 27 19-47 (1989).

[36] A.J. Short, S. Wehner, New J. Phys. 12033023 (2010).

[37] O. Bratteli and D.W. Robinson, Operator algebras and quantum statistical mechanics 1, Springer-Verlag, New York, 1987; O. Bratteli and D.W. Robinson, Operator algebras and quantum statistical mechanics 2, Springer-Verlag, Berlin, 1997.

[38] E. Alfsen, Compact Convex Sets and Boundary Integrals, Springer-Verlag, Berlin Heidelberg, New York, 1971.

[39] R. Phelps, Lectures on Choquet's Theorem, Springer-Verlag Berlin Heidelberg, (2001).

[40] W. Arveson and R. Kadison, arXiv:math/0508482 (2005).

[41] Y. Li and P. Busch, Journal of Mathematical Analysis and Applications Volume 408, 384-393, (2013).

[42] C. P. Niculescu and L.E. Persson, Convex Functions and their Applications. A Contemporary Approach. CMS Books in Mathematics 23, Springer Verlag, (2006).

[43] H. Halvorson and M. Müger, Algebraic quantum field theory, in Butterfield J and Earman J, editors, Philosophy of Physics, pp. 731-922, Elsevier, 2006.

[44] F. Holik, C. Massri, A. Plastino, and L. Zuberman, Int. J. Theor. Phys. 52 1836-1876 (2013).

[45] A. Khrennikov, Ubiquitous Quantum Structure-From Psychology to Finance, Springer, 2010.

[46] M. Rédei, and S.J. Summers, Stud. Hist. Philos. Sci. B 38 390-417 (2007).

[47] J. Yngvason, Rep. Math. Phys. 55 135-147 (2005). 
[48] B. Mielnik, Commun. Math. Phys. 9 55-80 (1968); B. Mielnik, Commun. Math. Phys. 15 1-46 (1969); B. Mielnik, Commun. Math. Phys. 3 221-256 (1974).

[49] F. Holik, C. Massri and N. Ciancaglini, Int. J. Theor. Phys. 51 1600-1620 (2012).

[50] F. Holik, C. Massri, A. Plastino, and L. Zuberman., Int. J. Theor. Phys. 52 1836-1876 (2013).

[51] G. Ludwig, Foundations of Quantum Mechanics I, Springer, Berlin-Heidelberg, Germany, 1983; G. Ludwig, Foundations of Quantum Mechanics II, Springer, Berlin-Heidelberg, Germany, 1985.

[52] Z. Daróczy, Inf. Control 1636 (1970).

[53] J. Havrda and F. Charvát, Kybernetika 330 (1967).

[54] P.N. Rathie, Inf. Sci. 5423 (1991).

[55] A.M. Gleason, J. Math. Mech. 6 885-893 (1957). 